\title{
Summary and Conclusion: Framing a New Research Agenda on Maternal Morbidities and Mortality in the United States
}

\author{
Michael C. Lu, MD, MS, MPH ${ }^{1}$ and Samia Noursi, $\mathrm{PhD}^{2}$
}

\begin{abstract}
Maternal mortality in the United States is at an alarming rate. Research can have an important role in addressing maternal mortality, but our current understanding of its causes and prevention remains woefully incomplete. The collection of articles in this volume begin to frame a new research agenda by asking four critical questions. First, what truly makes vulnerable populations vulnerable? Second, how do we prevent pregnancy complications and their long-term sequelae? Third, how can we make maternity care safer for all women? Finally, what can we do about the social, structural, and environmental determinants of maternal health? Answers to these questions can help inform practice, systems, and policy change to reduce and ultimately eradicate maternal deaths in the United States.
\end{abstract}

Keywords: maternal mortality, research agenda, vulnerable populations, prevention, safety bundle, social determinant

\section{Introduction}

N THE 21st CENTURY United States, no woman should ever die from pregnancy and childbirth. ${ }^{1}$ Yet each year, more than 700 mothers die, and more than 50,000 mothers experience a life-threatening complication (severe maternal morbidity). ${ }^{2}$ The maternal mortality rate in the United States has increased by $58 \%$ since 1990, a time during which 157 of 183 countries in a World Health Organization study reported significant declines in maternal death. ${ }^{3,4}$ Meanwhile, large racial, ethnic, socioeconomic, and geographic disparities persist. For example, African American women are more than thrice as likely to die from pregnancy and childbirth as White women, a gap that has not narrowed in decades. ${ }^{1}$

We can do better, and research has an important role to play. Research can help inform practice, systems, and policy change to reduce and ultimately eradicate maternal death in the United States. However, our current understanding of the causes and prevention of maternal mortality (and especially of the disparities) remains woefully incomplete. The collec- tion of articles in this volume summarizes the state of the science on maternal mortality. More important, they call out major knowledge gaps in four key areas: (i) vulnerable populations; (ii) prevention of pregnancy complications and their long-term sequelae; (iii) quality and safety of maternity care; and (iv) social, structural, and environmental determinants of maternal health. Collectively, these authors begin to sketch out a research agenda to guide the next generation of research on maternal morbidities and mortality.

\section{Vulnerable Populations}

Several articles ${ }^{5-10}$ in this special issue focus on vulnerable populations that are at greater risk of maternal morbidities and mortality. How little we know about some vulnerable populations is troubling. For example, Heck et al. ${ }^{5}$ deplore the dearth of reliable data on the causes of increased mortality risks among American Indian/Alaska Native women, especially for pregnancy-associated deaths. They suspect that homicide, suicide, and substance abuse and misuse-all linked

\footnotetext{
${ }^{1}$ Berkeley School of Public Health, University of California, Berkeley, Berkeley, California, USA.

${ }^{2}$ Office of Research on Women's Health, Office of the Director, National Institutes of Health, Bethesda, Maryland, USA.

(C) Michael C. Lu and Samia Noursi 2021; Published by Mary Ann Liebert, Inc. This Open Access article is distributed under the terms of the Creative Commons Attribution Noncommercial License (http://creativecommons.org/licenses/by-nc/4.0/) which permits any noncommercial use, distribution, and reproduction in any medium, provided the original author(s) and the source are cited.

Correction added on February 19, 2021 after first online publication of November 19, 2020: The article reflects Open Access, with copyright transferring to the author(s), and a Creative Commons Attribution Noncommercial License (CC-BY-NC) added (http:// creativecommons.org/licenses/by-nc/4.0/).
} 
to intimate partner violence-may play a role, but note that "severe data limitations prohibit our understanding of true incidence and prevalence." 5 Similarly, Signore et al. ${ }^{6}$ observe that "[d]espite the size of the disability population, the sexual and reproductive health needs of this population largely have been ignored." Available information on reproductive and pregnancy outcomes among women with disabilities suggests increased risks, especially as they relate to infection or cardiovascular conditions, some of which may be related to prepregnancy health status and social determinants, but much remains unknown. Patel and Sweeney ${ }^{7}$ raise concerns about the paucity of research on the reproductive health of transgender and gender nonconforming patients, who may face a whole host of health issues and risks from fertility treatments (which necessitate stoppage of gender-affirming hormone therapy), pregnancy, delivery, and lactation, including postpartum depression from gender dysphoria for transgender men. Similarly, Brown et al. ${ }^{8}$ pointed out that "[d] espite the wealth of research on calcium, iron, and folate and their effects on women's health outcomes, most studies conducted in the United States either included only White women or, when mixed ethnic/racial groups were included, the studies lacked adequate representation of specific groups that would allow appropriate subgroup analyses."

Even for populations that have been studied more extensively, such as women of advanced maternal age ${ }^{9}$ or African American women, ${ }^{10}$ much remains unknown about the root causes of their increased morbidity and mortality risk. Chinn et al. ${ }^{10}$ discuss how various social and historical factors-including slavery, institutionalized racism, and gender discrimination-shape the distinct social experience of Black womanhood in America. They posit that the accumulation of these stressors across the life course and generations can take a physiologic toll ("allostatic load") on the health of African American women and that such "weathering" could put them at greater risk for chronic health conditions, as well as maternal mortality and morbidities. Although theoretically compelling, ${ }^{11,12}$ empiric evidence linking "weathering" and "allostatic load" to maternal morbidities and mortality remains scant and inconclusive.

Taken together, these articles call for a greater focus on vulnerable populations in future research. First, we need to review, report, and learn from every maternal death (and every severe maternal morbidity), but especially those that occur among vulnerable populations. Current efforts by the Centers for Disease Control and Prevention to expand maternal mortality reviews to all 50 states are a good start. ${ }^{13}$ Second, we need to understand better what truly makes vulnerable populations vulnerable, which means future research needs to dive deeper into the root causes of their increased vulnerability. Third and most important, we need to learn what works in preventing maternal morbidities and mortality for vulnerable populations. This is a complex problem that will require bold, innovative, and complex solutions.

\section{Prevention of Pregnancy Complications and Their Long-Term Sequelae}

Despite significant progress in the past decades, several articles in this volume continue to find critical knowledge gaps in our understanding of the causes and prevention of a whole range of pregnancy complications, including hypertensive disorders of pregnancy, ${ }^{14}$ peripartum cardiomyopathy, ${ }^{14}$ gestational dia- betes, ${ }^{15}$ infectious diseases ranging from influenza to intraamniotic infections, ${ }^{16}$ and perinatal depression. ${ }^{17}$ Reducing and ultimately eradicating maternal mortality will require us to get a lot better at preventing pregnancy complications.

First, we need to get a lot better at primary preventionpreventing pregnancy complications from happening in the first place. Prevention requires better elucidation of predisease pathways leading up to the complications, as well as identification of effective interventions preconceptionally and in early pregnancy. As Varagic et al. ${ }^{14}$ point out, "there is growing recognition that the occurrence of pregnancy complications reliably identifies women with underlying, often unrecognized [cardiovascular] risk factors who may benefit from screening and preventive actions, such as preconception counseling to address risk and prepregnancy control of blood pressure, blood glucose, lipids, and weight." A number of established (e.g., high sensitivity C-reactive protein, triglycerides, lipids, glucose) and novel cardiovascular risk biomarkers (e.g., soluble fms-like tyrosine kinase 1, placental growth factor, vascular endothelial growth factor, endoglin) have been found to be altered in early pregnancy in women who later develop preeclampsia or peripartum cardiomyopathy, yet their utility in the primary prevention of cardiovascular complications in pregnancy remains to be shown. ${ }^{14}$ Similarly, Laposky and Pemberton ${ }^{18}$ identify sleep disordered breathing as a "potential therapeutic strategy to prevent or mitigate the progression of cardiometabolic disease across gestation, resulting in improved perinatal, postpartum, and future health in a substantial number of women," but acknowledge the need to improve clinical screening and identify effective interventions to reduce the risk of adverse cardiovascular and neonatal outcomes in pregnancy. Presently there is a dearth of proven effective primary prevention strategies for most pregnancy complications; one such proven intervention - the flu vaccine-is being increasingly undermined by vaccine hesitancy.

Second, we need to get a lot better at secondary preventiondetecting and intervening early in the course of a pregnancy complication. Eppes et al. ${ }^{19}$ discuss findings from state maternal mortality reviews, which showed that most maternal deaths in the United States are preventable and that delayed diagnosis and treatment of pregnancy complications play an important role. They highlight a study that found that implementing a Maternal Early Warning Trigger Tool, aimed at early detection and intervention of sepsis, cardiopulmonary dysfunction, hypertension-preeclampsia, and hemorrhage, led to a significant reduction in severe maternal morbidities over a 14 -month study period. ${ }^{20}$ More research on early warnings is needed. We also need more research on effective early interventions, including increased drug safety testing in pregnancy and lactation, as well as development of novel therapeutics targeting specific pathogenic mechanisms of pregnancy complications, such as sepsis or peripartum cardiomyopathy, before they spiral out of control. ${ }^{14,16}$ Dagher et al. ${ }^{17}$ discuss the problems of underdiagnosis and undertreatment of perinatal depression and the need to expand available treatment options ranging from interpersonal psychotherapy, cognitive behavioral therapy, and pharmacologic interventions to complementary and integrative health approaches- these may include folate supplementation, yoga, acupuncture, and mindfulness, although the evidence for their full efficacy and safety remains limited. Thomas et al. ${ }^{21}$ also call for more research to better address pain during and after pregnancy. 
Third, we need to get a lot better at tertiary preventionpreventing the serious and long-term sequelae of pregnancy complications. Silva et al. ${ }^{15}$ call attention to the "effects of maternal dysglycemia on mothers later in life and the transgenerational cycle of metabolic disease risk rooted in maternal dysglycemia." Similarly, Varagic et al. ${ }^{14}$ observe that women's long-term cardiovascular health could be linked to adverse pregnancy outcomes. They review several large cohort studies and meta-analyses that found pregnancy complications-such as preeclampsia and other hypertensive disorders of pregnancy, gestational diabetes, preterm birth, pregnancy loss, and small for gestational age-to be associated with long-term risk for cardiovascular diseases in women. ${ }^{14}$ In light of the growing body of evidence supporting both pregnancy as a window to a woman's future health outlooks and intergenerational transmission of health risks to the offspring, an urgent need exists to identify effective interventions for tertiary prevention of the long term and perhaps intergenerational sequelae of pregnancy complications.

\section{Quality and Safety of Maternity Care}

A promising area for reducing maternal morbidities and mortality is making maternity care safer. More than half of maternal deaths in the United States are preventable ${ }^{22}$; many result from clinician, facility, and system factors, such as inadequate training, missed or delayed diagnosis, delayed or ineffective response, and poor communication and coordination among clinicians. ${ }^{23}$

Since 2013, the Council on Patient Safety in Women's Health Care and the Alliance for Innovation on Maternal Health program has developed 10 patient safety bundles designed to improve the quality and safety of maternity care. $^{24}$ These safety bundles "contribute to improved outcomes by ensuring reliably delivered evidenced-based care; promoting team collaboration; and enabling organizations and health care teams to systematically approach a redesign of their processes through bundle implementation in order to improve the reliability of care processes." ${ }^{25}$ In this volume, Eppes et al. ${ }^{19}$ review evidence of the effectiveness of obstetric hemorrhage and severe hypertension bundles for addressing maternal early warning signs, highlighting the work of the California Perinatal Quality Care Collaborative. Implementation of the postpartum hemorrhage bundlewhich focused on improving readiness (e.g., postpartum hemorrhage cart), recognition (e.g., quantitative measurement of blood loss), response (e.g., early uterotonic use), and reporting-resulted in a $20.8 \%$ reduction in severe maternal morbidities, compared to a $1.2 \%$ reduction at control hospitals. ${ }^{26}$ Similarly, implementation of the severe hypertension bundle led to a significant increase in compliance with treatment recommendations, from $50 \%$ to $>90 \%$, as well as a decrease in the incidence of eclampsia by $42.6 \%$ and severe maternal morbidities by $16.7 \% .^{27}$ These improvements in California led to the launch of the Alliance for Innovation in Maternal Health by the federal Maternal and Child Health Bureau in 2015. Led by the American College of Obstetricians and Gynecologists and in collaboration with 25 national organizations, the Alliance has engaged 33 states and more than 1400 hospitals in implementing these maternal safety bundles. $^{28}$ This work needs to be expanded nationwide and rigorously evaluated.

\section{Social, Structural, and Environmental Determinants of Maternal Health}

Several articles in this volume focused on the social, structural, and environmental determinants of maternal health. Campbell et al. ${ }^{29}$ explore the interface between intimate partner violence and pregnancy-associated and pregnancy-related deaths due to homicide, suicide, and drug overdose. Boyles et al. ${ }^{30}$ review the literature on the role chemical and nonchemical environmental stressors have on maternal health. Environmental stressors are associated with a variety of immediate maternal health impacts, including hypertensive disorders of pregnancy, fibroids, and infertility, as well as long-term maternal health impacts, such as higher risk of breast cancer and metabolic disorders. ${ }^{30}$ Saluja and Bryant $^{31}$ call attention to the potential role of implicit bias on patient-provider communication and its potential impact on maternal health care and outcomes. Crear-Perry et al. ${ }^{32}$ call out racism as a structural determinant and root cause of inequities in maternal health outcomes, noting that "many of the social and political structures and policies in the United States were born out of racism, classism, and gender oppression."

A major unanswered question and the Holy Grail for a new research agenda on maternal morbidities and mortality in the United States is, "What can we do about social, structural, and environmental determinants of maternal health?" The collection of articles in this volume makes a number of recommendations, including assuring traumainformed health care for pregnant women, ${ }^{29}$ strengthening implicit bias and cultural humility training for health care providers and trainees, ${ }^{31}$ integrating data on social determinants of health into electronic health records and clinical decision support, ${ }^{33}$ and capturing environmental exposures across diverse racial and ethnic populations in big data initiatives, such as the National Institutes of Health's All of Us Research Program. ${ }^{30}$

Tackling social, structural, and environmental inequities underlying maternal health disparities will require more than developing new services, programs, and trainings; it will require effecting real social, political, and policy change. Crear-Perry et al. ${ }^{32}$ offer several policy solutions that may help advance health equity, defined as "assurance of the conditions for optimal health for all people by valuing all populations equally, recognizing and rectifying historical injustices, and providing resources according to need," including guaranteeing paid family leave; expanding access to and scope of health insurance coverage; assuring respectful, culturally appropriate care; and incentivizing community investments. Research can help inform and evaluate societal, political, and policy changes. Such changes will not be easy or clean but achieving health equity will require no less.

\section{Conclusion}

Decreasing the high rates of maternal mortality in the United States is an achievable goal. Research has an important role to play in reducing and ultimately eradicating maternal mortality and changing the status quo.

The collection of articles in this volume begins to lay out a new research agenda by asking several important unanswered research questions. First, what truly makes vulnerable 
populations vulnerable? Second, how do we prevent pregnancy complications and their long-term sequelae? Third, how can we make maternity care safer for all women? Last, what can we do about the social, structural, and environmental determinants of maternal health? Given that maternal mortality is one of the most sensitive indicators of population health, answers to these questions may help inform a more enlightened national strategy, not only for preventing maternal morbidities and mortality but also for improving the health of our nation.

\section{Author Disclosure Statement}

No competing financial interests exist.

\section{Funding Information}

No funding was received for this article.

\section{References}

1. Lu MC. Reducing maternal mortality in the United States. JAMA 2018;320:1237-1238.

2. Centers for Disease Control and Prevention. Pregnancy mortality surveillance system. Available at: https://cdc.gov/ reproductivehealth/maternalinfanthealth/pmss.html Accessed July 9, 2020.

3. Douthard RA, Martin IK, Chapple-McGruder T, Langer A, Chang S. U.S. maternal mortality within a global context: Historical trends, current state, and future directions. J Womens Health 2021;30:168-177.

4. WHO, UNICEF, UNFPA, World Bank, United Nations Population Division. Trends in maternal mortality: 1990 to 2013, 2014. Available at: http://who.int/reproductivehealth/ publications/monitoring/maternal-mortality-2013/en/ Accessed July 9, 2020.

5. Heck JL, Jones EJ, Bohn D, et al. Maternal mortality among American Indian/Alaska Native women: A scoping review. J Womens Health 2021;30:220-229.

6. Signore C, Davis M, Tingen CM, Cernich AN. The intersection of disability and pregnancy: Risks for maternal morbidity and mortality. J Womens Health 2021;30:147153.

7. Patel S, Sweeney LB. Maternal health in the transgender population. J Womens Health 2021;30:253-259.

8. Brown LL, Cohen BE, Edwards E, Gustin CE, Noreen Z. Physiological need for calcium, iron, and folic acid for women of various subpopulations during pregnancy and beyond. J Womens Health 2021;30:207-211.

9. Correa-de-Araujo R, Yoon S. Clinical outcomes in highrisk pregnancies due to advanced maternal age. J Womens Health 2021;30:160-167.

10. Chinn JJ, Martin K, Redmond N. Health equity among Black women in the United States. J Womens Health 2021; 30:212-219.

11. Geronimus AT. The weathering hypothesis and the health of African-American women and infants: Evidence and speculations. Ethn Dis 1992;2:207-221.

12. Lu MC, Halfon N. Racial-ethnic disparities in birth outcomes: A life-course perspective. Matern Child Health J 2003;7:13-30.

13. Centers for Disease Control and Prevention. Enhancing reviews and surveillance to eliminate maternal mortality
(ERASE MM). Available at: https://cdc.gov/reproductive health/maternal-mortality/erase-mm/index.html Accessed July 9, 2020.

14. Varagic J, Desvigne-Nickens P, Gamble-George J, et al Maternal morbidity and mortality: Are we getting to the "heart" of the matter? J Womens Health 2021;30:178-186.

15. Silva CM, Arnegard ME, Maric-Bilkan C. Dysglycemia in pregnancy and maternal-fetal outcomes. J Womens Health 2021;30:187-193.

16. PrabhuDas M, Piper JM, Jean-Philippe P, LachowiczScroggins M. Immune regulation, maternal infection, vaccination, and pregnancy outcome. J Womens Health 2021; 30:199-206.

17. Dagher RK, Bruckheim HE, Colpe LJ, Edwards E, White DB. Perinatal depression: Challenges and opportunities. J Womens Health 2021;30:154-159.

18. Laposky AD, Pemberton VL. Sleep-disordered breathing and pregnancy-related cardiovascular disease. J Womens Health 2021;30:194-198.

19. Eppes CS, Han SB, Haddock AJ, et al. Enhancing obstetric safety through best practices. J Womens Health 2021;30: 265-269.

20. Shields LE, Wiesner S, Klein C, Pelletreau B, Hedriana HL. Use of maternal early warning trigger tool reduces maternal morbidity. Am J Obstet Gynecol 2016;214: 527.e1-527.e6.

21. Thomas DA, Bruckheim HE, White JM. The need to consider pregnancy as a biological variable to reduce preventable suffering related to pregnancy. J Womens Health (Larchmt) 2020;X:pp-pp.

22. Davis NL, Smoots AN, Goodman DA. Pregnancy-related deaths: Data from 14 u.s. maternal mortality review committees, 2008-2017. Atlanta, GA: Centers for Disease Control and Prevention, U.S. Department of Health and Human Services, 2019. Available at: https://cdc.gov/reproductive health/maternal-mortality/erase-mm/MMR-Data-Brief_2019h.pdf Accessed July 9, 2020.

23. Review to Action. Building U.S. capacity to review and prevent maternal deaths. Report from nine MMRCs, 2018. Available at: http://reviewtoaction.org/Report_from_Nine_ MMRCs Accessed July 9, 2020.

24. Council on Patient Safety in Women's Health Care. Alliance for innovation on maternal health (AIM). Available at: http://safehealthcareforeverywoman.org/aim-program Accessed July 9, 2020.

25. Resar R, Griffin FA, Haraden C, Nolan TW. Using care bundles to improve health care quality. IHI innovation series white paper, 2012. Available at: http://ihi.org/resources/ Pages/IHIWhitePapers/UsingCareBundles.aspx Accessed July $9,2020$.

26. Main EK, Cape V, Abreo A, Vasher J, Woods A, Carpenter A, Gould JB. Reduction of severe maternal morbidity from hemorrhage using a state perinatal quality collaborative. Am J Obstet Gynecol 2017;216:298.e1-298.e11.

27. Shields LE, Wiesner S, Klein C, Pelletreau B, Hedriana HL. Early standardized treatment of critical blood pressure elevations is associated with a reduction in eclampsia and severe maternal morbidity. Am J Obstet Gynecol 2017;216: 415.e1-415.e5.

28. Maternal and Child Health Bureau. Alliance for innovations in maternal health. Available at: https://mchb.hrsa.gov/ maternal-child-health-topics/maternal-and-womens-health Accessed July 9, 2020. 
29. Campbell J, Matoff-Stepp S, Velez ML, Cox HH, Laughon K. Pregnancy-associated deaths from homicide, suicide, and drug overdose: Review of research and the intersection with intimate partner violence. J Womens Health 2021;30: 236-244.

30. Boyles AL, Beverly BE, Fenton SE, et al. Environmental factors involved in maternal morbidity and mortality. J Womens Health 2021;30:245-252.

31. Saluja B, Bryant Z. How implicit bias contributes to racial disparities in maternal morbidity and mortality in the United States. J Womens Health 2021;30:270273.

32. Crear-Perry J, Correa-de-Araujo R, Lewis Johnson T, McLemore MR, Neilson E, Wallace M. Social and structural determinants of health inequities in maternal health. J Womens Health 2021;30:230-235.
33. Jean-Francois B, Lash TB, Dagher RK, Green Parker MC, Han SB, Lewis Johnson T. The potential for health information technology tools to reduce racial disparities in maternal morbidity and mortality. J Womens Health 2021; 30:274-279.

Address correspondence to: Michael C. Lu, MD, MS, MPH Berkeley School of Public Health University of California, Berkeley 2121 Berkeley Way Berkeley, CA 94720-1670 USA

E-mail: mclu@berkeley.edu 